\title{
Evaluation of Patient Satisfaction and Factors Affecting It: A Review of the Literature
}

\author{
Fereshteh Farzianpour*, Raziye Byravan*, Sara Amirian \\ Health Care Management, Department of Health Management and Economics, School of Public Health, \\ Tehran University of Medical Sciences, Tehran, Iran \\ Email: *farzianp@sina.tums.ac, Beyranvand.raziye@yahoo.com
}

Received 12 September 2015; accepted 9 November 2015; published 12 November 2015

Copyright (C) 2015 by authors and Scientific Research Publishing Inc.

This work is licensed under the Creative Commons Attribution International License (CC BY). http://creativecommons.org/licenses/by/4.0/

c) (7) Open Access

\section{Abstract}

Patient satisfaction with hospital services is one of the most important indicators of effectiveness and quality of hospital services. Patient satisfaction surveys can provide valuable data for evaluating the current status, awareness of quality and quantity of process improvement programs and quality improvement to health managers and policy makers. The present study discusses previous studies conducted in this area, and presents recommendations to improve patient satisfaction. The method of this study is review. Selected papers were identified by searching keyword in Persian and English in different databases and after applying the inclusion criteria, 19 studies were extracted and analyzed. 23,793 patients in 12 studies entered into this research were investigated. Generally, satisfaction of the patients in this study was estimated at an average level. Continuous evaluation of patient satisfaction and identification of the factors affecting it requires establishment of comprehensive and accurate data system in this area; that by application of the results of previous studies steps can be taken to improve patient satisfaction. Patients' satisfaction in Iran, compared with other countries, is desirable. In addition, the overall satisfaction of patients in recent years has increased compared to previous years and the reason can be measures like different ways of improving the quality, customer orientation, and applying the results of research conducted in this area.

\section{Keywords}

Evaluation, Patient Satisfaction, Factors Affecting Satisfaction

${ }^{*}$ Corresponding authors. 


\section{Introduction}

Patient satisfaction is a concept that has received great attention today in health care and it is one of the main goals and priorities of the health and treatment system. Any system needs awareness and use of customers and clients' opinion to improve its efficiency. Since patient is the main customer of services of hospital, his satisfaction can somehow show proper provision of services, therefore patients are valuable information resource for judging the quality of care and to be able to interpret quality of care, acceptance rate of patients and their expectations should be recognized since the most reliable way to assess the quality and quantity of health care services is access to patients' views [1].

Patient satisfaction is a cognitive response that is influenced by various factors. Determining factors affecting satisfaction, is considered an indirect way to achieve real patient satisfaction, in this regard, evaluating patient satisfaction by determining factors affecting satisfaction and understanding expectations and needs of patients and eliminating dissatisfaction causes can enhance service provision level and as a result increase satisfaction of patients and improvement of their physical and mental health will be better and rapid; and the aim of health system which is promotion of care quality is achieved. Assessment of this index can provide valuable data for evaluating the current status, awareness of quality and quantity of process improvement programs and quality improvement to health managers and policy makers [2]. In the competitive world of health care, importance of patient satisfaction and his quality of life is increasingly considered [3]. The importance of this issue in the health care system is getting more because experience of illness and the need for compliance and follow-up treatment and care process increase patients' vulnerability and their need to comprehensive support [4]. In addition, satisfaction is one of the aspects of individuals' health and if health system does not pay attention to patient satisfaction, it has acted contrary to its mission which is responding needs of people for health [4]. Satisfaction is the outcome a complex set of different factors and in order to achieve that it is necessary to coordinated various aspects of services such as nursing, medicine, support and various organizational sections in some way and with full respect for patients' rights in all aspects, create appropriate conditions for its improvement [4].

Several studies have been conducted in different countries and have investigated patient satisfaction from different points of view. These points of view include how patient has access to services, having knowledge of patient admission, first encounters of health center with patient wait time to hospitalization of patient, treatment of medical personnel, including doctors and nurses, way of patient costs payment, etc. And even in a study the role and importance of welcoming as first meeting with patient while admission to hospital is investigated and notes that appropriate initial treatment at the hospital helps eliminating stress and fear and increase patient satisfaction of hospital [5].

According to what mentioned and search database, we found that a large number of studies with different sample sizes and different hypotheses have been performed on measurement of patient satisfaction that if the results are paid attention to deeply, valuable information is obtained. Accordingly, we do a review in this area, it is hoped that the results of this study will be useful.

\section{Methods}

The present study aimed to evaluate patient satisfaction from hospital services through review method and search in domestic and international websites. Search in resources has been done by the following main keyword:

Patient satisfaction, Hospital, In-patient satisfaction, Quality of health services, Factors associated with patient satisfaction

Factors considered in the selection of articles include:

- Articles must have been published from 2008 onwards

- Their main areas of discussion is related to patient satisfaction and factors affecting satisfaction of hospitalized patient in hospital

- Full-text of the articles is available in the mentioned databases.

- Studies are real in terms of actual sample size.

- Studies that had just measured patient satisfaction with merely one variable such as level of education, type of insurance, gender and so on, are not selected.

Among the papers found in the literature, after limiting the number of searches, 3310 ones had almost our considered factors and among them some were removed because of factors such as the subject's being repeti- 
tious or unclear methodology and finally 12 articles in Persian and 7 was ones in English were selected for this review study. After determining the relevant studies in terms of titles and content, note taking was done from title of each article, the first author's name, year of publication, sample size, purpose of study, methodology, results and findings of them to record the required information in the selected studies.

\section{Findings}

By searching keywords in resources referred to in methods section and after applying the inclusion criteria, 16 qualified articles (12 articles in Persian and 7 ones in Latin) were selected (Figure 1, Flowchart 1).

In total, in these 19 articles, sample was 23,493 people. Eight cases of the studies under review reported overall sample size, and did not divide it by sex and in most of these studies patient satisfaction has not been reported by division of gender. The method of 12 studies is cross-sectional and 7 ones descriptive. Sampling was simple random in 12 cases, non-random in 3 cases and targeted in 4 studies. Among the studies entered this study, the highest percentage of patients satisfaction was for the study by Ebrahimi and et al. [6] that took place in 2012 on 696 patients, in this survey patient satisfaction was reported higher than 98\%. Also, the lowest percentage of patients satisfaction was related to the study by Karimi [7] conducted in 2013 on 196 patients. Great inconsistency between the results of studies under review was observed that might be due to years of study, place of study (Iran or other countries) or size of selected samples. Percent overall satisfaction of patients in each study along with the study year, first author's name and the sample size are brought Table 1 for ease of comparison and analysis.

Salami et al. in a study in 2010 concluded that $86 \%$ of patients were satisfied with the provided service and about $50 \%$ said that if they get ill again, they will refer to that hospital for treatment. In this study relationship hospital personnel with patient was known as the most important factor in patient satisfaction [8]. Sajadian et al. in a study in 2008 concluded that 5 variables of study including education level, reference type, dealing with lack of empty beds, employment status and gender have significant correlation with patients' satisfaction and after that it was found that the variable educational level has more powerful impact on patient satisfaction [9]. Heydari et al. in a study in 2008 concluded that two factors have key roles in patient satisfaction and increase it including how the doctor and nurse treat the patient, hospital cleanliness and reducing patient waiting time [10].

The study by Oyvind Bjertnaes et al. 2011, 2013 and Press in BMJ shows that variables such as age and gender have little effect on satisfaction. But treatment of nursing staff, physicians and waiting time for appointments are key elements affecting patient's satisfaction [11] [12]. Nohi et al. in a study showed that the lowest rate of complete satisfaction is related to accounting and the greatest satisfaction was related to nurses themselves. Dissatisfaction about facilities was significantly higher than other areas [13]. Soleimanpour et al. in a study concluded that the following factors have a key role in patient satisfaction: timely presence of nurse, speed of admission and filing process, improving facilities, nutritional status and handling patient. It was also found

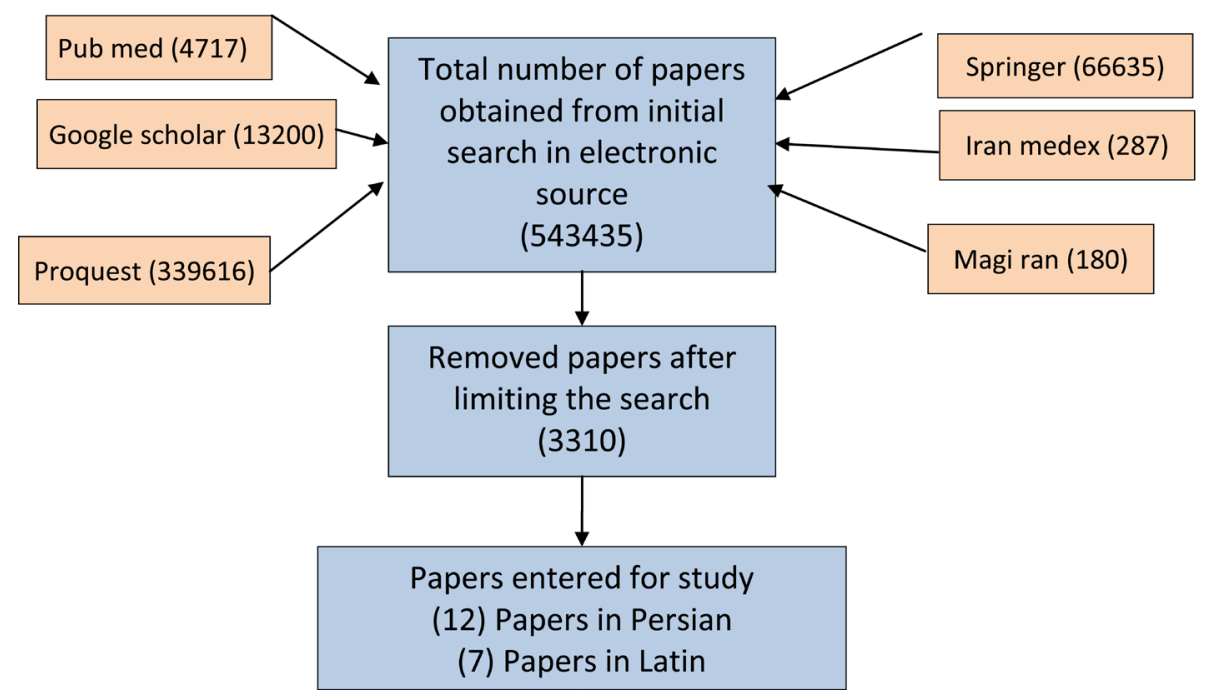

Figure 1. Flowchart of review and search for articles. 
Table 1. Percent of patient satisfaction of hospital services received by division of entered studies.

\begin{tabular}{cccccc}
\hline Title & First author & Sample size & Place of study & Publication year & Percent of patient satisfaction \\
\hline 1 & Salami et al. & 350 & Isfahan & 2012 & 86.6 \\
2 & Heydari et al. & 360 & QOM & 2008 & 64.4 \\
3 & Solaymanpour et al. & 303 & Tabriz & 2008 & 62 \\
4 & Ebrahim iet al. & 696 & Entire country of Iran & 2012 & 98 \\
5 & Farzianpour et al. & 320 & Tehran & 2010 & 53 \\
6 & Ebrahimi & 196 & Urmia & 2012 & 134 \\
7 & Karimi et al. & 120 & Isfahan & 2013 & 78 \\
8 & Salami et al. & 400 & Isfahan & 2012 & 187 \\
9 & Sajadian et al. & 100 & Tehran & 2008 & 56.6 \\
10 & Heydari et al. & 300 & Qom & 2008 & 80.5 \\
11 & Oyvind et al. & 10,912 & Norway & 2013 & 80 \\
12 & Oyvind et al. & 10,912 & Norway & 2011 & 87 \\
13 & Nohi et al. & 302 & Kerman & 2009 & 65 \\
14 & Soleimanpour et al. & 360 & Tabriz & 2011 & 85 \\
15 & Skudal et al. & 500 & Nordic & 2012 & 76 \\
16 & Riiskjær et al. & 244 & Dubai & 2012 & 80 \\
17 & Lagu et al. & 500 & America & 2009 & 76 \\
18 & Santuzzi et al. & 360 & England & 2009 & 85 \\
\hline 19 & Peters & 244 & Germany & 2010 & 80 \\
\hline
\end{tabular}

that factors such as age, education, type of insurance coverage affect patient satisfaction [14]. The study by Skudal et al. in four Nordic countries showed that the least patient satisfaction was related to administrative and financial units and the highest satisfaction was from public and welfare services.

Also determining factors for dissatisfaction and attempt to solve them and identify and correct some hospital processes, especially the processes of acceptance and discharge are among cases that in addition to making it possible to provide services with higher quality, leads to patient satisfaction [15]. Riiskjær et al. in a study in 2012 observed strong positive correlation between the index of hospital environment and patient satisfaction. There is also a strong correlation between patient satisfaction and a feeling of health that shows crucial role of treatment staff in attracting satisfaction of and patients [16].

Britnas et al. in a study in 2011 concluded that 13 factors affect patients' overall satisfaction. The most important factor influencing patient satisfaction was the experience regarding nursing services and then expectations of patients, patient experience of the doctor visit and understanding false treatment. In this study, survey of patients determined whether patient expectations are consistent with the service received or not, and finally according to the suggestions of patient, measure are taken to improve status of hospital [11].

The study by Lagu et al. on hospitals of England University of Medical Sciences revealed that 5 variables are strongly associated with patient satisfaction including: marital status, education, type of hospital, unit of hospitalization, and cost to be paid. It was also found that there is significant relationship between satisfaction and individual characteristics such as age, education, occupation and marital status [17].

\section{Discussion}

In recent years, several studies have been conducted in the field of patient satisfaction from hospital services and since a fundamental element of providing service in a society is satisfaction of clients is, therefore, attention to this issue and monitoring is completely justifiable [17]. Because behavior of customer and consumer (patient) is a sign of quality of service, satisfaction rate and ultimately identifying strengths and weaknesses in service delivery, so investigating views of consumers and the factors affecting their satisfaction and taking measures in line with these affecting factors can be effective in enhancing patient satisfaction index [18]. 
This study was attempted to present a clear picture of overall satisfaction of patients and the main factors affecting by organized review and summarizing recent studies although the results of different studies in this field shows great inconsistency. In this study, factors such as age, sex, marital status, education level, social status of the patient, the waiting time for services, skill of hospital staff, services provided by doctors and nurses, providing instructions to patients while discharge, respect for the views of patients, insurance status of the patient and so on have been recognized as effective on satisfaction of patients from hospital services [18].

The survey showed that patient satisfaction is on average level, on the other hand given that this study has investigated and compared the papers in Iran and abroad, this comparison may not seem logical and cause bias, so in this study it is tried that among foreign studies, those are selected that the place of study is similar to Iran as much as possible. In addition, it should be noted that patient satisfaction in Iran and mentioned countries cannot be judged absolutely just based on numbers reported from studies, but an introductory judgmental can be achieved from these results [18].

Satisfaction measuring studies with low sample volume unconsciously have bias in sampling from more satisfied patients and this removes the opportunity to investigate group of people who have not enough satisfaction with the service provided but have valuable information for managers and planners of health centers. Therefore, in investigations performed on patient satisfaction, ensuring a representative sample size and use of appropriate sampling method are among basic necessities of studies on patient satisfaction [19].

Some of the factors that have been identified as factors affecting patient satisfaction in the studies reviewed include:

- Treatment result

- Gender

- Age group

- Education level

- Marital status

- Manner of hospital management

- Facilities (housekeeping)

- Behavior of the staff and doctor

- Medical insurance

- Processes of administrative and financial units

- Speed of admission and filing

- Food Quality

- Feeling healthy

According to the results obtained in this study, the following key factors have been identified as effective in improving patient satisfaction:

- Awareness of managers in the field of patient satisfaction

- Planning to improve hospital status

- Infrastructure needed to make appropriate decisions in order to optimize hospital services

- Informing about the trend of patient treatment

- Determining the causes of dissatisfaction and attempt to fix them

- Identification and modification of some correctable medical procedures such as discharge and acceptance process

- Development of insurance services in addition to providing optimum services

- Paying more attention to accurate and comprehensive insurance coverage

- Increasing force and equipping hospitals and using specialists

- Providing more amenities, preparing food with appropriate quality and quantity

- Monitoring the performance of nurses and hospital cleanliness

- Devoting more time to visit the patient

- Further strengthening the referral system and providing services to people in the form of referral system

\section{Conclusions}

Nevertheless, no study can fully determine and describe all the factors affecting patient satisfaction in detail, also according to investigations conducted in this study, there are conflicting results. For example, some studies recognize demographic characteristics of patient effective on satisfaction, while others have considered this factor 
as ineffective.

In general it can be said that with the strengthening information system, to find the causes of dissatisfaction and attempt to resolve the shortcomings and proper planning to provide better service with high quality, necessary context for improvement of patient satisfaction can be provided.

\section{References}

[1] Bjertnæs, Ø.A., Sjetne, I.S. and Iversen, H.H. (2012) Overall Patient Satisfaction with Hospitals: Effects of PatientReported Experiences and Fulfilment of Expectations. BMJ Quality \& Safety, 21, 39-46. http://dx.doi.org/10.1136/bmjqs-2011-000137

[2] Grøndahl, V.A. (2012) Patients’ Perceptions of Actual Care Conditions and Patient Satisfaction with Care Quality in Hospital. Karlstad University Studies: Dissertation, Karlstad University, Karlstad.

[3] Farzianpour, F. and Godarzi, L. (2010) Evaluation of Customer Relationship Management (CRM) in a Teaching Hospital Affiliated with Tehran University of Medical Sciences. Tehran University, Tehran.

[4] Sjetne, I.S., Bjertnæs, Ø.A., Olsen, R.V., Iversen, H.H. and Bukholm, G. (2011) The Generic Short Patient Experiences Questionnaire (GS-PEQ): Identification of Core Items from a Survey in Norway. BMC Health Services Research, 11, 88. http://dx.doi.org/10.1186/1472-6963-11-88

[5] Peters, E., Dieckmann, N. and Dixon, A. (2010) Less Is More in Presenting Quality Information to Consumers. Medical Care Research and Review, 64, 169-190. http://dx.doi.org/10.1177/10775587070640020301

[6] Ebrahimi Rigi Tanha, Z., Bagaee, R. and Feizi, A. (2012) A Survey of Frequency and Type of Medical Errors Done by Nursing Students of Urmia Medical Sciences University in 2011. The Journal of Urmia Nursing and Midwifery Faculty, 10, 139-143.

[7] Karimi, S., Masoodian, Y., Yaghoubi, M., Hosseini, M. and Sadeghifar, J. (2013) Patient Satisfaction with Provided Services and Facilities in Educational Hospitals of Isfahan, Iran, from Admission to Discharge. Health Information Management, 9, 785.

[8] Salami, S. and Samouei, R. (2012) Assessment of Patient Satisfaction from Health Services in Educational Hospitals of Isfahan, Iran. Health Information Management, 8, 1103.

[9] Sajadian, A., Kaviani, A., Yonesian, M. and Montazeri, A. (2008) Patient Satisfaction: A Descriptive Study of Breast Care Clinic in Iran. Iranian Journal of Breast Diseases, 1, 32-44.

[10] Heydari, A. and Seydi, M. (2008) Patient Satisfaction of General Practitioners in the City of Qom and Its Influencing Factors 2005- 2006. Journal of Medical Council of Islamic Republic of Iran, 26, 530-540.

[11] Bjertnaes, O., Skudal, K.E. and Iversen, H.H. (2013) Classification of Patients Based on Their Evaluation of Hospital Outcomes: Cluster Analysis Following a National Survey in Norway. BMC Health Services Research, 13, 73. http://dx.doi.org/10.1186/1472-6963-13-73

[12] Bjertnaes, O., Skudal, K.E. and Iversen, H.H. (2011) Overall Patient Satisfaction with Hospitals: Effects of PatientReported Experiences and Fulfillment of Expectations. BMJ Quality \& Safety, 21, 39-46. http://dx.doi.org/10.1136/bmjqs-2011-000137

[13] Nohi, E. and Poraboli B. (2009) Patient Discharge and Satisfaction of Educational Needs of Practice Nurses in Kerman. Hormozgan Medical Journal, 13, 206-212.

[14] Soleimanpour, H., Gholipouri, C., Salarilak, S., Raoufi, P., Vahidi, R.G., Rouhi, A.J., et al. (2011) Emergency Department Patient Satisfaction Survey in Imam Reza Hospital, Tabriz, Iran. International Journal of Emergency Medicine, 4, 21-29. http://dx.doi.org/10.1186/1865-1380-4-2

[15] Skudal, K.E., Garratt, A., Eriksson, B., et al. (2012) The Nordic Patient Experiences Questionnaire (NORPEQ): Cross-National Comparison of Data Quality, Internal Consistency, and Validity in Four Nordic Countries. BMJ Open, 2, e000854. http://dx.doi.org/10.1136/bmjopen-2012-000864

[16] Riiskjær, E., Ammentorp, J. and Kofoed, P.E. (2012) The Value of Open-Ended Questions in Surveys on Patient Experience: Number of Comments and Perceived Usefulness from a Hospital Perspective. International Journal for Quality in Health Care, 24, 509-516. http://dx.doi.org/10.1093/intqhc/mzs039

[17] Lagu, T., Goff, S.L., Hannon, N.S., et al. (2013) A Mixed-Methods Analysis of Patient Reviews of Hospital Care in England: Implications for Public Reporting of Health Care Quality Data in the United States. Joint Commission Journal on Quality and Patient Safety, 39, 7-15.

[18] Santuzzi, N.R., Brodnik, M.S., Rinehart-Thompson, L., et al. (2009) Patient Satisfaction: How Do Qualitative Comments Relate to Quantitative Scores on a Satisfaction Survey? Quality Management in Health Care, 18, 3-18. http://dx.doi.org/10.1097/01.QMH.0000344589.61971.41

[19] Peters, E., Dieckmann, N. and Dixon, A. (2010) Less Is More in Presenting Quality Information to Consumers. Medical Care Research and Review, 64, 169-190. http://dx.doi.org/10.1177/10775587070640020301 